Research Article

\title{
Computing the dependence of graph energy on nullity: The method of siblings
}

\author{
Ivan Gutman* \\ Faculty of Science, University of Kragujevac, 34000 Kragujevac, Serbia
}

(Received: 11 April 2021. Accepted: 6 May 2021. Published online: 7 May 2021.)

(C) 2021 the author. This is an open access article under the CC BY (International 4.0) license (www.creativecommons.org/licenses/by/4.0/).

\begin{abstract}
The energy of a graph is the sum of absolute values of its eigenvalues. The nullity of a graph is the algebraic multiplicity of number zero in its spectrum. The dependence of graph energy on nullity is of importance in chemical applications. In this paper we present a method by means of which this dependence can be calculated.
\end{abstract}

Keywords: energy (of graph); nullity (of graph); spectrum (of graph).

2020 Mathematics Subject Classification: 05C50, 05C92.

\section{Introduction}

Let $G$ be a simple graph, possessing $n$ vertices, and let $\mathbf{A}(G)$ be its $(0,1)$-adjacency matrix of $G$ (a symmetric matrix of order $n$ ). The eigenvalues of $\mathbf{A}(G)$, denoted by $\lambda_{1} \geq \lambda_{2} \geq \cdots \geq \lambda_{n}$, form the spectrum of $G$ [2]. The number of eigenvalues of $G$ that are equal to zero, i.e., the algebraic multiplicity of the number zero, is the nullity of $G$, denoted by $\eta=\eta(G)$.

A graph with $\eta(G)>0$ is said to be singular. Otherwise, $G$ is non-singular.

The characteristic polynomial of the graph $G$ is defined as [2]

$$
\phi(G, x)=\operatorname{det}\left(x \mathbf{I}_{n}-\mathbf{A}(G)\right)
$$

where $\mathbf{I}_{n}$ is the unit matrix of order $n$. In coefficient-form this polynomial is written as

$$
\phi(G, x)=\sum_{k=0}^{n} c_{k} x^{n-k} .
$$

Note that all zeros of $\phi(G, x)$ are real-valued numbers (equal to the graph eigenvalues). In addition, if $G$ is singular, then $c_{n}=0$, whereas otherwise $c_{n} \neq 0$.

The energy of the graph $G$ is defined as [15]:

$$
E=E(G)=\sum_{i=1}^{n}\left|\lambda_{i}\right|
$$

As well known [11,15], the concept of graph energy is a generalization of the total $\pi$-electron energy [5,11]. In chemical applications, the graph nullity is the number of non-bonding molecular orbitals, and is closely related to the stability and chemical reactivity of the underlying molecule [3-5].

Already in the early days of the study of graph energy, it was observed that it somehow decreases with the increasing nullity [5,7]. In particular, if $G$ and $G^{*}$ are two structurally similar graphs, and if $G$ is non-singular whereas $G^{*}$ is singular, then one expects that $E(G)>E\left(G^{*}\right)$.

The problem is that the meaning of "structurally similar graphs" is not clear and - from a mathematical point of view cannot be satisfactorily defined. Earlier attempts to solve this problem were based on designing approximate expressions for $E(G)$, containing the term $\eta[7,12,13]$.

In a recent paper [9], a pseudo-spectral formalism was proposed for computing the energy-effect of nullity. According to it, the energy of the non-singular graph $G$ is calculated from its characteristic polynomial $\phi(G, x)$, which is the standard procedure. On the other hand, a quasi-energy $E^{*}(G)$ is calculated from a polynomial $\phi^{*}(G, x)$, constructed to be as similar as possible to $\phi(G, x)$, but having nullity $\eta>0$. This polynomial could be understood as the characteristic polynomial of a pseudo-graph $G^{*}$, which in the general case does not need to be a true graph. 
In [9], it was shown that the polynomial $\phi^{*}(G, x)$ should be defined as

$$
\phi^{*}(G, x)=\phi(G, x)-c_{n} .
$$

Then by applying the Coulson-Jacobs formula [1], one gets [9]

$$
E(G)-E^{*}(G)=\frac{2}{\pi} \int_{0}^{\infty} \ln \left|\frac{\phi(G, i x)}{\phi(G, i x)-c_{n}}\right| d x .
$$

The hidden difficulty in formula (2) is that the pseudo-spectrum of $G^{*}$ (i.e., the solutions of $\phi^{*}(G, x)=0$ ), may be complex-valued, and is anyway hard to be calculated. Consequently, in the general case, the evaluation of the right-hand side of Equation (2) is far from being straightforward.

In the next section, we show that there exists a special case in which the computation of the energy effect of nullity becomes almost trivially easy.

\section{Graph siblings}

Definition 2.1. Let $G$ be a non-singular simple graph and $G^{*}$ a singular simple graph. Let $\phi(G, x)$ and $\phi\left(G^{*}, x\right)$ be their characteristic polynomials. We say that $G$ and $G^{*}$ are siblings (or, more precisely: spectral siblings) if the difference $\phi(G, x)-$ $\phi\left(G^{*}, x\right)$ is independent of the variable $x$.

In Figure 1 are depicted the smallest pairs of graph siblings.

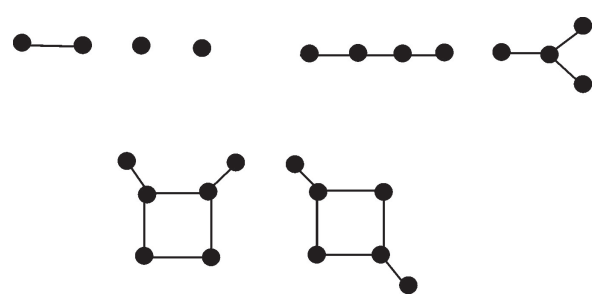

Figure 1: The smallest pairs of graph siblings: disconnected, trees, and cycle-containing. For instance, the characteristic polynomials of the 6 -vertex siblings are $x^{6}-6 x^{4}+4 x^{2}-1$ and $x^{6}-6 x^{4}+4 x^{2}$.

Directly from Definition 2.1, it follows that graph siblings must have equal number of vertices and, with the exception of the 2-vertex case, equal number of edges. In addition, we have:

Lemma 2.1. If the graphs $G$ and $G^{*}$ are siblings, then in parallel to Equation (1), it holds

$$
\phi\left(G^{*}, x\right)=\sum_{k=0}^{n-1} c_{k} x^{n-k}
$$

which means that $\phi(G, x)-\phi\left(G^{*}, x\right)=c_{n}$.

Combining Lemma 2.1 with Equation (2), we get:

Theorem 2.1. If the graphs $G$ and $G^{*}$ are siblings, then the effect of nullity on the energy of $G$ is equal to $E(G)-E\left(G^{*}\right)$.

Thus, in the case of siblings, the energy effect of nullity can be straightforwardly evaluated by calculating two ordinary graph energies.

Graph siblings are relatively easy to construct. In what follows, we demonstrate this for two such families depicted in Figure 2.

Lemma 2.2. The trees $T_{1}(k)$ and $T_{2}(k), k=1,2, \ldots$, are siblings.

Proof. Denote by $P_{n}$ the path on $n$ vertices.

If $u$ is a pendent vertex of a tree $T$, adjacent to the vertex $v$, then $[8,10,14]$

$$
\phi(T, x)=x \phi(T-u, x)-\phi(T-u-v, x)
$$

which applied to $P_{n}$ yields the well known recursion relation

$$
\phi\left(P_{n}, x\right)=x \phi\left(P_{n-1}, x\right)-\phi\left(P_{n-2}, x\right) .
$$




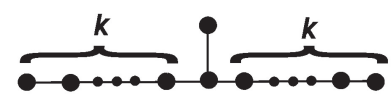

$T_{1}(k)$

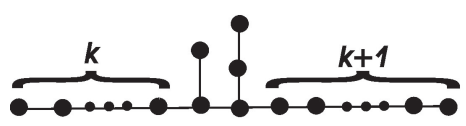

$T_{3}(k)$

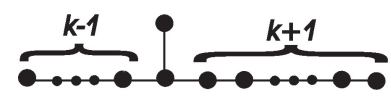

$T_{2}(k)$

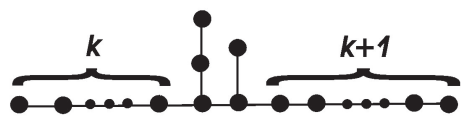

$T_{4}(k)$

Figure 2: Two families of tree siblings. $T_{1}(k)$ and $T_{3}(k)$ are non-singular for $k=2,4,6, \ldots$, in which case $T_{2}(k)$ and $T_{4}(k)$ are singular. For $k=1,3,5, \ldots, T_{2}(k)$ and $T_{4}(k)$ are non-singular, whereas $T_{1}(k)$ and $T_{3}(k)$ are singular.

From (4) it follows

$$
\begin{aligned}
& \phi\left(P_{n}, x\right) \phi\left(P_{n}, x\right)-\phi\left(P_{n-1}, x\right) \phi\left(P_{n+1}, x\right) \\
= & {\left[x \phi\left(P_{n-1}, x\right)-\phi\left(P_{n-2}, x\right)\right] \phi\left(P_{n}, x\right)-\phi\left(P_{n-1}, x\right)\left[x \phi\left(P_{n}, x\right)-\phi\left(P_{n-1}, x\right)\right] } \\
= & \phi\left(P_{n-1}, x\right) \phi\left(P_{n-1}, x\right)-\phi\left(P_{n-2}, x\right) \phi\left(P_{n}, x\right) \\
\cdots & \cdots \cdots \\
= & \phi\left(P_{n-\ell}, x\right) \phi\left(P_{n-\ell}, x\right)-\phi\left(P_{n-1-\ell}, x\right) \phi\left(P_{n+1-\ell}, x\right)
\end{aligned}
$$

which for $\ell=n-1$ gives

$$
\begin{aligned}
\phi\left(P_{n}, x\right) \phi\left(P_{n}, x\right)-\phi\left(P_{n-1}, x\right) \phi\left(P_{n+1}, x\right) & =\phi\left(P_{1}, x\right) \phi\left(P_{1}, x\right)-\phi\left(P_{0}, x\right) \phi\left(P_{2}, x\right) \\
& =x \cdot x-1 \cdot\left(x^{2}-1\right)
\end{aligned}
$$

i.e.,

$$
\phi\left(P_{n}, x\right) \phi\left(P_{n}, x\right)-\phi\left(P_{n-1}, x\right) \phi\left(P_{n+1}, x\right)=1 .
$$

Applying (3) to $T_{1}(k)$ and $T_{2}(k)$, we get

$$
\begin{aligned}
\phi\left(T_{1}(k), x\right) & =x \phi\left(P_{2 k+1}, x\right)-\phi\left(P_{k}, x\right) \phi\left(P_{k}, x\right) \\
\phi\left(T_{2}(k), x\right) & =x \phi\left(P_{2 k+1}, x\right)-\phi\left(P_{k-1}, x\right) \phi\left(P_{k+1}, x\right)
\end{aligned}
$$

from which, by identity (5),

$$
\phi\left(T_{1}(k), x\right)-\phi\left(T_{2}(k), x\right)=-\left[\phi\left(P_{k}, x\right) \phi\left(P_{k}, x\right)-\phi\left(P_{k-1}, x\right) \phi\left(P_{k+1}, x\right)\right]=-1 .
$$

Therefore $T_{1}(k)$ and $T_{2}(k)$ are siblings.

Remark 2.1. From the above proof it follows that the forests $P_{n} \cup P_{n}$ and $P_{n-1} \cup P_{n+1}$ are siblings for all $n \geq 1$.

Lemma 2.3. The trees $T_{3}(k)$ and $T_{4}(k), k=1,2, \ldots$, are siblings.

Proof. If $e$ is an edge of a tree $T$, connecting the vertices $u$ and $v$, then $[8,10,14]$

$$
\phi(T, x)=\phi(T-e, x)-\phi(T-u-v, x) .
$$

Applying (6) to the edges of $T_{3}(k)$ and $T_{4}(k)$, lying between the two branching vertices, we get

$$
\begin{aligned}
& \phi\left(T_{3}(k), x\right)=\phi\left(P_{k+2}, x\right) \phi\left(P_{k+4}, x\right)-\phi\left(P_{1}, x\right) \phi\left(P_{2}, x\right) \phi\left(P_{k}, x\right) \phi\left(P_{k+1}, x\right) \\
& \phi\left(T_{4}(k), x\right)=\phi\left(P_{k+3}, x\right) \phi\left(P_{k+3}, x\right)-\phi\left(P_{1}, x\right) \phi\left(P_{2}, x\right) \phi\left(P_{k}, x\right) \phi\left(P_{k+1}, x\right)
\end{aligned}
$$

from which, by identity (5),

$$
\phi\left(T_{3}(k), x\right)-\phi\left(T_{4}(k), x\right)=-\left[\phi\left(P_{k+3}, x\right) \phi\left(P_{k+3}, x\right)-\phi\left(P_{k+2}, x\right) \phi\left(P_{k+4}, x\right)\right]=-1 .
$$

Therefore $T_{3}(k)$ and $T_{4}(k)$ are siblings. 
In Table 1 we give the effects of nullity on the energy of the sibling trees depicted in Figure 2.

Table 1. Effect of nullity on the energies of the non-singular trees $T_{1}(k)$ and $T_{3}(k)$ for $k=2,4, \ldots, 10$, and of the nonsingular trees $T_{2}(k)$ and $T_{4}(k)$ for $k=1,3, \ldots, 9$, computed according to Theorem 2.1.

\begin{tabular}{||c|cc||c|cc||}
\hline$k$ & $T_{1}(k)$ & $T_{3}(k)$ & $k$ & $T_{2}(k)$ & $T_{4}(k)$ \\
\hline 2 & 0.74361 & 0.61117 & 1 & 1.00803 & 0.74361 \\
4 & 0.48809 & 0.42835 & 3 & 0.58083 & 0.49007 \\
6 & 0.36737 & 0.33244 & 5 & 0.41641 & 0.36888 \\
8 & 0.29566 & 0.27249 & 7 & 0.32635 & 0.29672 \\
10 & 0.24780 & 0.23122 & 9 & 0.26892 & 0.24855 \\
\hline
\end{tabular}

\section{References}

[1] C. A. Coulson, J. Jacobs, Conjugation across a single bond, J. Chem. Soc. (1949) 2805-2812.

[2] D. Cvetković, M. Doob, H. Sachs, Spectra of Graphs - Theory and Application, Academic Press, New York, 1980.

[3] D. M. Cvetković, I. Gutman, The algebraic multiplicity of the number zero in the spectrum of a bipartite graph, Mat. Vesnik (Beograd) 9 (1972) $141-150$.

[4] D. Cvetković, I. Gutman, N. Trinajstić, Graphical studies on the relations between the structure and reactivity of conjugated systems: The role of non-bonding molecular orbitals, J. Mol. Struct. 28 (1975) 289-303.

[5] A. Graovac, I. Gutman, N. Trinajstić, Topological Approach to the Chemistry of Conjugated Molecules, Springer, Berlin, 1977.

[6] I. Gutman, Acyclic systems with extremal Hückel $\pi$-electron energy, Theor. Chim. Acta 45 (1977) 79-87.

[7] I. Gutman, Bounds for total $\pi$-electron energy of conjugated hydrocarbons, Z. Phys. Chem. (Leipzig) 266 (1985) 59-64.

[8] I. Gutman, A survey on the matching polynomial, In: Y. Shi, M. Dehmer, X. Li, I. Gutman (Eds.), Graph Polynomials, CRC Press, Boca Raton, 2016, pp. 77-99.

[9] I. Gutman, Graph energy and nullity, Open J. Discrete Appl. Math. 4 (2021) 25-28.

[10] I. Gutman, H. Hosoya, On the calculation of the acyclic polynomial, Theor. Chim. Acta 48 (1978) 279-286.

[11] I. Gutman, O. E. Polansky, Mathematical Concepts in Organic Chemistry, Springer, Berlin, 1986.

[12] I. Gutman, S Radenković, S. Đorđević, I. Milovanović, E. Milovanović, Total $\pi$-electron and HOMO energy, Chem. Phys. Lett. 649 (2016) $148-150$.

[13] I. Gutman, S. Radenković, S. Đorđević, I. Milovanović, E. Milovanović, Extending the McClelland formula for total $\pi$-electron energy, J. Math. Chem. $\mathbf{5 5}$ (2017) 1934-1940.

[14] H. Hosoya, Graphical enumeration of the coefficients of the secular polynomials of the Hückel molecular orbitals, Theor. Chim. Acta 25 (1972) $215-222$.

[15] X. Li, Y. Shi, I. Gutman, Graph Energy, Springer, New York, 2012. 